\title{
Safety of Patients with Hepatitis C Virus Treated with Glecaprevir/Pibrentasvir from Clinical Trials and Real-World Cohorts
}

\author{
Xavier Forns · Jordan J. Feld · Douglas E. Dylla · Stanislas Pol · Kazuaki Chayama • \\ Jinlin Hou · Jeong Heo · Pietro Lampertico • Ashley Brown • Mark Bondin • Fernando Tatsch • \\ Margaret Burroughs · John Marcinak · Zhenzhen Zhang · Amanda Emmett · Stuart C. Gordon • \\ Ira M. Jacobson
}

Received: March 9, 2021 / Accepted: April 18, 2021 / Published online: May 22, 2021

(C) The Author(s) 2021

\section{ABSTRACT}

Introduction: More than 70 million people are estimated to be infected with hepatitis $C$ virus (HCV) globally. If left untreated, HCV infection can lead to complications such as extensive liver fibrosis, cirrhosis, and hepatocellular carcinoma (HCC). Evolution of treatments has resulted in highly effective and well-tolerated

Supplementary Information The online version contains supplementary material available at https:// doi.org/10.1007/s12325-021-01753-3.

X. Forns $(\bowtie)$

Liver Unit, Hospital Clinic de Barcelona, IDIBAPS and CIBEREHD, University of Barcelona, Barcelona, Spain

e-mail: xforns@clinic.cat

J. J. Feld

Toronto Centre for Liver Disease, University Health Network, University of Toronto, Toronto, ON, Canada

D. E. Dylla - M. Bondin · F. Tatsch - M. Burroughs . J. Marcinak · Z. Zhang · A. Emmett

AbbVie Inc., North Chicago, IL, USA

S. Pol

Liver Unit, Cochin Hospital, APHP, Inserm U-1223, Institut Pasteur, Université de Paris, Paris, France

K. Chayama

Department of Gastroenterology and Metabolism, Graduate School of Biomedical and Health Sciences, Hiroshima University, Hiroshima, Japan all-oral direct-acting antivirals. The pangenotypic regimen of glecaprevir/pibrentasvir is approved for treating HCV for patients without cirrhosis or with compensated cirrhosis (CC). Guidelines have evolved to simplify treatment to enable non-specialists to manage and treat HCV-infected patients. Simultaneously, such treatment algorithms provide guidance on the pretreatment identification of small subsets of patients who may require specialist treatment and long-term follow-up for advanced liver disease, including those at risk of developing HCC. This study describes the safety profile of glecaprevir/pibrentasvir in patients identified

\section{J. Hou}

Department of Infectious Diseases, State Key Laboratory of Organ Failure Research, Guangdong Provincial Key Laboratory of Viral Hepatitis Research, Nanfang Hospital, Southern Medical University, Guangzhou, China

\section{J. Heo}

Department of Internal Medicine, College of Medicine, Pusan National University and Medical Research Institute, Busan, Republic of Korea

\section{P. Lampertico}

Division of Gastroenterology and Hepatology, CRC "A.M. and A. Migliavacca" Center for Liver Disease, Foundation IRCCS Ca' Granda Ospedale Maggiore Policlinico, Milan, Italy

\section{P. Lampertico}

Department of Pathophysiology and

Transplantation, University of Milan, Milan, Italy 
using previously described noninvasive laboratory measures who may be eligible for treatment by non-liver specialists.

Methods: This post hoc analysis of glecaprevir/ pibrentasvir in patients, identified by noninvasive laboratory measures, intended to exclude patients with advanced liver disease and severe renal impairment, who can be managed within non-liver specialist settings. Patients were included from clinical trials and real-world studies of glecaprevir/pibrentasvir for $\mathrm{HCV}$ treatment. Baseline demographics, clinical characteristics, and safety assessments, including adverse events and laboratory abnormalities, were summarized.

Results: Data across these large-scale studies confirm that glecaprevir/pibrentasvir is well tolerated across different patient populations, with fewer than $0.1 \%$ of patients experiencing a serious adverse event related to treatment drugs, and few patients developing HCC during or after treatment.

Conclusion: The safety profile of glecaprevir/ pibrentasvir enhances the confidence of nonliver specialists to treat the majority of HCVinfected patients, and provides an opportunity to expand the treater pool, potentially increasing diagnosis and treatment rates for $\mathrm{HCV}$, contributing to elimination of $\mathrm{HCV}$.

Keywords: Hepatitis C virus; Non-specialist; Safety

A. Brown

Imperial College Healthcare NHS Trust, London, UK

S. C. Gordon

Division of Gastroenterology and Hepatology,

Henry Ford Health System and Wayne State

University School of Medicine, Detroit, MI, USA

I. M. Jacobson

NYU Langone Health, New York, USA

\section{Key Summary Points}

Why carry out this study?

Simplification of the hepatitis $C$ virus (HCV) care cascade and the advent of direct-acting antivirals can help to achieve the World Health Organization's (WHO) $2030 \mathrm{HCV}$ elimination targets.

Guidelines have attempted to simplify treatment and enable non-liver specialists to manage and treat low-risk HCVinfected patients, who represent the majority of patients. However, non-liver specialists may have concerns over treatment safety by using simplified, noninvasive pretreatment assessments.

This study investigated the safety profile of glecaprevir/pibrentasvir in patients identified using previously described noninvasive laboratory measures who may be eligible for treatment by non-liver specialists.

\section{What was learned from the study?}

Data from large clinical trials and realworld studies included in this analysis confirm that glecaprevir/pibrentasvir is well tolerated across different patient populations, with fewer than $0.1 \%$ of patients experiencing a serious adverse event related to treatment drugs, and no significant hepatoxicity observed.

The safety profile of glecaprevir/ pibrentasvir may provide clinical confidence to physicians and other nonliver specialists treating HCV with the opportunity to expand the treater pool, a necessary step to meet HCV elimination targets. 


\section{DIGITAL FEATURES}

This article is published with digital features, including a summary slide, to facilitate understanding of the article. To view digital features for this article go to https://doi.org/10.6084/ m9.figshare.14438756.

\section{INTRODUCTION}

Hepatitis $\mathrm{C}$ virus (HCV) infection is one of the leading causes of chronic liver disease worldwide. If left untreated, HCV can lead to hepatic scarring (fibrosis), hepatic cirrhosis (extensive scarring), and increase risk for developing hepatocellular carcinoma (HCC) [1]. HCV treatment is curative, defined as sustained virologic response (SVR; unquantifiable HCV ribonucleic acid) 12 weeks after treatment completion. HCV cure prevents the development of cirrhosis and consequently is associated with reducing risk of liver-related mortality, clinical decompensation, and HCC development compared to untreated patients [1-4]. Successful HCV therapy can reduce risk of extrahepatic complications, such as cardiovascular disorders [5] and diabetes [6]. Owing to the availability of highly effective treatment, HCV elimination is possible, with the World Health Organization (WHO) aiming to reduce new viral hepatitis infections by $90 \%$ and deaths due to viral hepatitis by $65 \%$ by 2030 [7].

Pretreatment assessment guidelines in several countries, including the USA, France, Australia, and Spain, have evolved to guide nonliver specialists such as nurse practitioners and general practitioners in the management of $\mathrm{HCV}$, thus increasing treatment capacity [8-13]. Guideline bodies have recently released simplified treatment algorithms that may facilitate wider treatment acceptance within primary care settings, thus reducing care cascade gaps [10]. These algorithms help identify easy-to-treat patients with less advanced liver disease or at low risk for negative liver-related outcomes. For example, the American Association for the Study of Liver Disease (AASLD) recommends simplified pretreatment assessments using noninvasive, serological, or imaging-based techniques for assessing liver fibrosis [10]. These guidelines recommend identifying patients with the likelihood of cirrhosis using Fibrosis-4 score (FIB-4) calculated by the following noninvasive measures: age, aspartate aminotransferase (AST), platelet count, and alanine aminotransferase (ALT) [10]. A FIB- $4>3.25$ is suggestive of advanced liver disease and cirrhosis [10]. Another well-accepted noninvasive measure to identify patients at low risk for developing complications was identified by the Baveno VI Consensus Workshop combining FibroScan ${ }^{\circledR}$ (Echosens, Waltham, MA) $<20 \mathrm{kPa}$ and a corresponding platelet count $\geq 150 \times$ $10^{9} / \mathrm{L}$ [14]. An investigational assessment for identifying patients with a low risk of liver-related outcomes during direct-acting antiviral (DAA) treatment is a combination of albu$\min >38 \mathrm{~g} / \mathrm{L}$, indicating normal synthetic function, [15] and platelet count $\geq 130 \times 10^{9} /$ $\mathrm{L}$. The aforementioned laboratory measures are feasible in primary care settings [16-18] and can identify patients without decompensated cirrhosis who could be suitable for treatment by non-liver specialists.

With the simplified pretreatment assessments, non-liver specialists' concerns may include treatment safety and effectiveness. Glecaprevir/pibrentasvir (G/P) is a fixed-dose, once-daily, all-oral combination DAA therapy approved to treat all common genotypes of chronic HCV in patients without cirrhosis or with compensated cirrhosis (CC) [19, 20]. Clinical trials have shown G/P to be well tolerated and highly effective, even in patients with CC, with an overall cure rate of 98\% [21]. In light of such data, AASLD also recommends simplified 8-week G/P treatment for some patients with CC without requirement for baseline resistance testing [10].

The present analysis was conducted to describe the safety profile of G/P in patients identified using previously described noninvasive laboratory measures who may be eligible for treatment by non-liver specialists. 


\section{METHODS}

\section{Study Design}

Two separate data analyses were performed using data from real-world post-marketing observational studies (PMOS) enrolling patients from Austria, Belgium, France, Greece, Israel, Italy, Poland, Portugal, and Switzerland and pooled data from the following $\mathrm{G} / \mathrm{P}$ clinical trials: ENDURANCE-1 (NCT02604017), ENDURANCE-2 (active arms) (NCT02640482), ENDURANCE-3 (NCT02640157), ENDURANCE-4 (NCT02636595), ENDURANCE56 (NCT02966795), EXPEDITION-1 (NCT02642432), EXPEDITION-2 (NCTO2738138), EXPEDITION-3 (NCT03219216), EXPEDITION-8 (NCT03089944), VOYAGE-1 (active arms) (NCT03222583), VOYAGE-2 (NCT03235349), CERTAIN-1 (NCT02707952), CERTAIN-2 (NCT02723084), SURVEYOR-2 (parts 3 and 4) (NCT02243293), APRI (NCT03212521), and MAGELLAN-1 (part 1 arm C, part 2 arms D, E) (NCT02446717). All patients received 300/120 mg G/P taken once daily with food for 8,12 , or 16 weeks. Patients in the clinical trials were followed for 24 weeks posttreatment, and in the PMOS cohorts SVR12 data were collected. For all included studies, written informed consent was obtained from each patient, including consent to participate and permission to publish personal health information while ensuring the identity of the individual remains confidential. For all included studies, the study protocols conformed to the ethical guidelines of the 1964 Declaration of Helsinki and its later updates, and were approved by the appropriate institutional review boards.

\section{Patient Population}

For these analyses, any patients with severe renal impairment, defined as chronic kidney disease stage $4 / 5$, were excluded. Eligibility criteria for the originating studies have been previously reported. In addition, patients with cirrhosis underwent HCC screening within 3 months prior to screening of the originating studies either by ultrasound, computed tomography scan, or magnetic resonance imaging, or had a negative ultrasound at screening. Patients with HCV genotype 1-6, without cirrhosis or with CC, treatment-naïve or experienced to interferon or pegylated interferon \pm ribavirin, or sofosbuvir + ribavirin \pm pegylated interferon, and human immunodeficiency viruscoinfected patients, were included in these analyses.

Patients potentially eligible for HCV treatment in non-liver specialist settings were identified using common noninvasive methods. Nonmutually exclusive patient subgroups were based on baseline laboratory measures of advanced liver disease and transient elastography:

1. Baseline FIB- $4<3.25$ [22].

2. Baseline FibroScan $<20 \mathrm{kPa}$ and platelet count $\geq 150 \times 10^{9} / \mathrm{L}[14]$.

3. Baseline albumin $>38 \mathrm{~g} / \mathrm{L}$ and platelet count $\geq 130 \times 10^{9} / \mathrm{L}$.

4. Patients who met at least one of the prior criteria [10].

\section{Endpoints and Assessments}

Baseline demographics, clinical characteristics, and safety assessments, including adverse events (AE) and laboratory abnormalities, were summarized. AEs were assessed by a study investigator for a possible relationship to $G / P$, and were coded using Medical Dictionary for Regulatory Authorities (MedDRA) 22.1 [23]. Treatment-emergent AEs (defined as any AE that occurred after the first dose of $G / P$ and within 30 days after the last dose of $G / P$ ) of special interest for hepatic decompensation or hepatic failure and treatment-emergent and non-treatment-emergent AEs of special interest for HCC were also assessed using MedDRA 22.1 [23] preferred terms. Baseline and maximum laboratory values were cross tabulated to calculate rates of normalization. SVR was also summarized.

\section{Statistical Analysis}

Safety results and demographic and baseline characteristics were summarized for the intentto-treat population, which included all patients 
who received at least one dose of G/P. Categorical variables were summarized by number and percentage.

\section{RESULTS}

As a result of overlap in patients defining the three individual subgroups (baseline FIB$4<3.25$, baseline FibroScan $<20 \mathrm{kPa}$ and platelet count $\geq 150 \times 10^{9} / \mathrm{L}$, and baseline albu$\min >38 \mathrm{~g} / \mathrm{L}$ and platelet count $\geq 130 \times 10^{9} /$ $\mathrm{L})$, the results described in the text are those of the overall subgroups of patients who met at least one of the prior criteria for both clinical trial and PMOS cohorts, unless stated otherwise.

\section{Patient Characteristics}

Demographics and baseline characteristics are reported for all clinical trials and PMOS subgroups determined by the noninvasive test criteria (Table 1). The FibroScan $<20 \mathrm{kPa}$ and platelet count $\geq 150 \times 10^{9} / \mathrm{L}$ patient subgroup was smaller than other subgroups for both cohorts because not all patients had baseline FibroScan conducted. For patients who met at least one of the serum test criteria, $14.2 \%$ and $6.4 \%$ of the clinical trial cohort and PMOS cohort had CC at baseline, respectively. Realworld utilization of 8-week treatment duration $(89.0 \%)$ in the PMOS population accurately reflects $\mathrm{HCV}$ patient populations at the time data were collected. In the clinical trial cohort, $74.6 \%(2802 / 3754)$ of patients were taking concomitant medications; those taken by at least $5 \%$ of patients were paracetamol, ibuprofen, acetylsalicylic acid, amlodipine, and levothyroxine. In the PMOS cohort, $46.9 \%$ $(634 / 1352)$ of patients were reported as taking concomitant medications, which included levothyroxine $(3.9 \%)$, methadone $(3.9 \%)$, and acetylsalicylic acid (3.8\%).

\section{Efficacy}

SVR12 rates were at least $97.5 \%$ in the intent-totreat clinical trial cohort and at least $97.6 \%$ in the PMOS core population with sufficient follow-up with no meaningful differences among the four subgroups (Fig. 1).

\section{Safety}

AEs, laboratory parameters, and laboratory parameter abnormalities were collected (Table 2).

Overall, in the clinical trial cohort $60.6 \%$ (2275/3754) experienced an $\mathrm{AE}$, while $31.5 \%$ $(1184 / 3754)$ and $0.3 \%(10 / 3754)$ of patients experienced an AE possibly related to the study drug and an AE leading to treatment discontinuation, respectively. Most common AEs were headache (495/3754 [13.2\%]), fatigue (377/3754 [10.0\%]), and nausea (254/3754 [6.8\%]) in this cohort. Most common AEs leading to treatment discontinuation were angioedema (2/3754 $[<0.1 \%])$, anxiety $(2 / 3754[<0.1 \%])$, and nausea $(2 / 3754[<0.1 \%])$. Serious AEs experienced by at least two patients in the clinical trial cohort were joint dislocation $(n=3)$ and angina unstable, angioedema, bile duct stone, bronchitis, gastric ulcer, and transient ischemic attack (all $n=2$ ).

In the clinical trial cohort, $5(0.1 \%)$ patients reported HCC during or after treatment (Supplementary Material). One $(<0.1 \%)$ patient experienced a treatment-emergent hepatic decompensation event of worsening ascites. This patient with decompensated cirrhosis had moderate ascites present at study screening by ultrasound but not recognized at that time, and therefore was considered a protocol violation. This patient continued G/P treatment without interruption and achieved SVR without additional worsening of symptoms. This patient was included in this analysis on the basis of a baseline FIB-4 of 3.05, but did not qualify for other subgroup analyses because of a baseline FibroScan of $26.3 \mathrm{kPa}$, platelet count of $114 \times 10^{9} / \mathrm{L}$, and albumin of $27 \mathrm{~g} / \mathrm{L}$. This patient also had a FibroTest (BioPredictive, Paris, France) of 0.97, indicating cirrhosis.

In the PMOS cohort, $13.8 \%(187 / 1352)$ of patients experienced an AE, with $7.8 \%$ (106/ $1352)$ and $0.4 \%(6 / 1352)$ of patients experiencing an AE possibly related to the study drug and an $\mathrm{AE}$ leading to treatment discontinuation, 


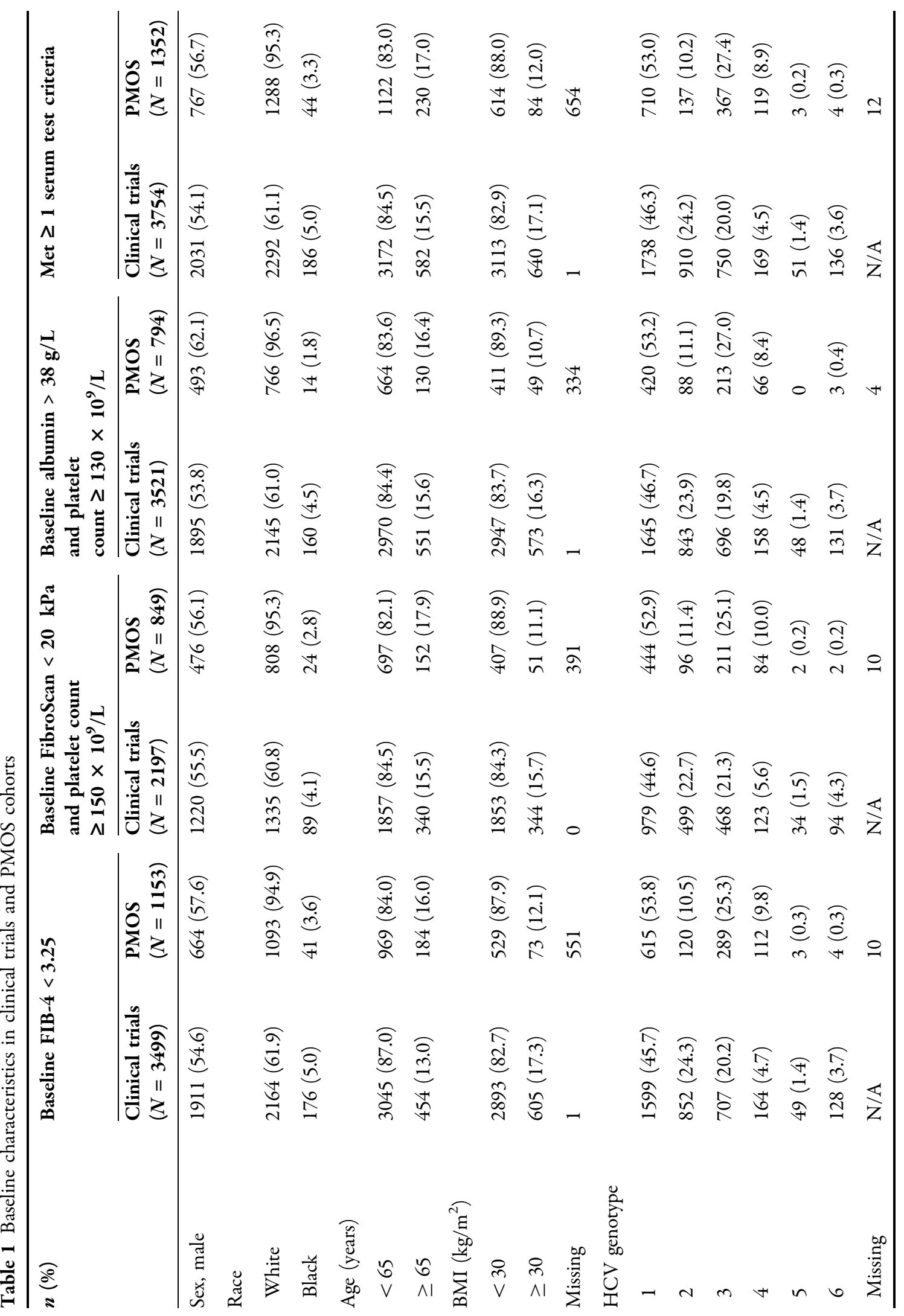




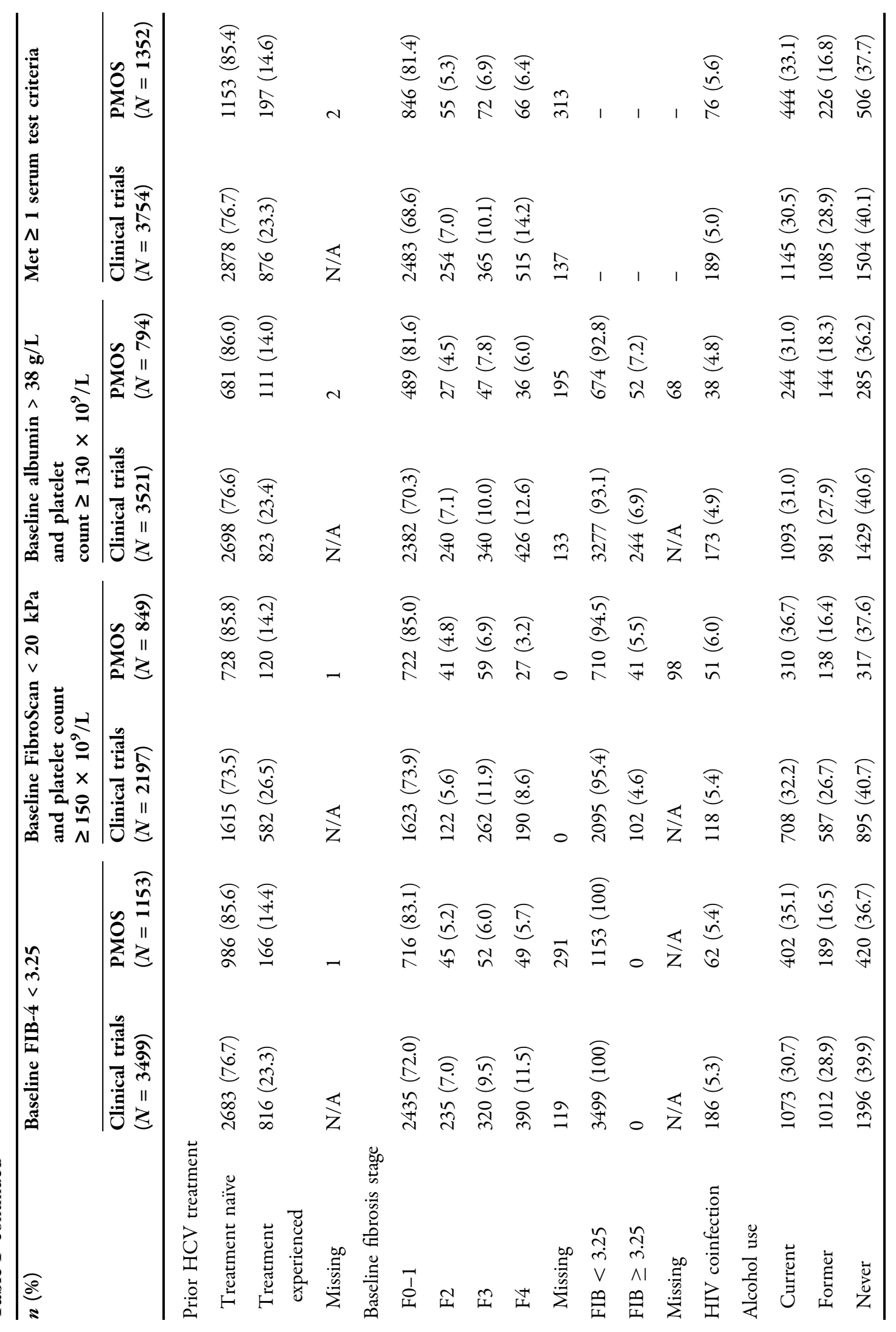




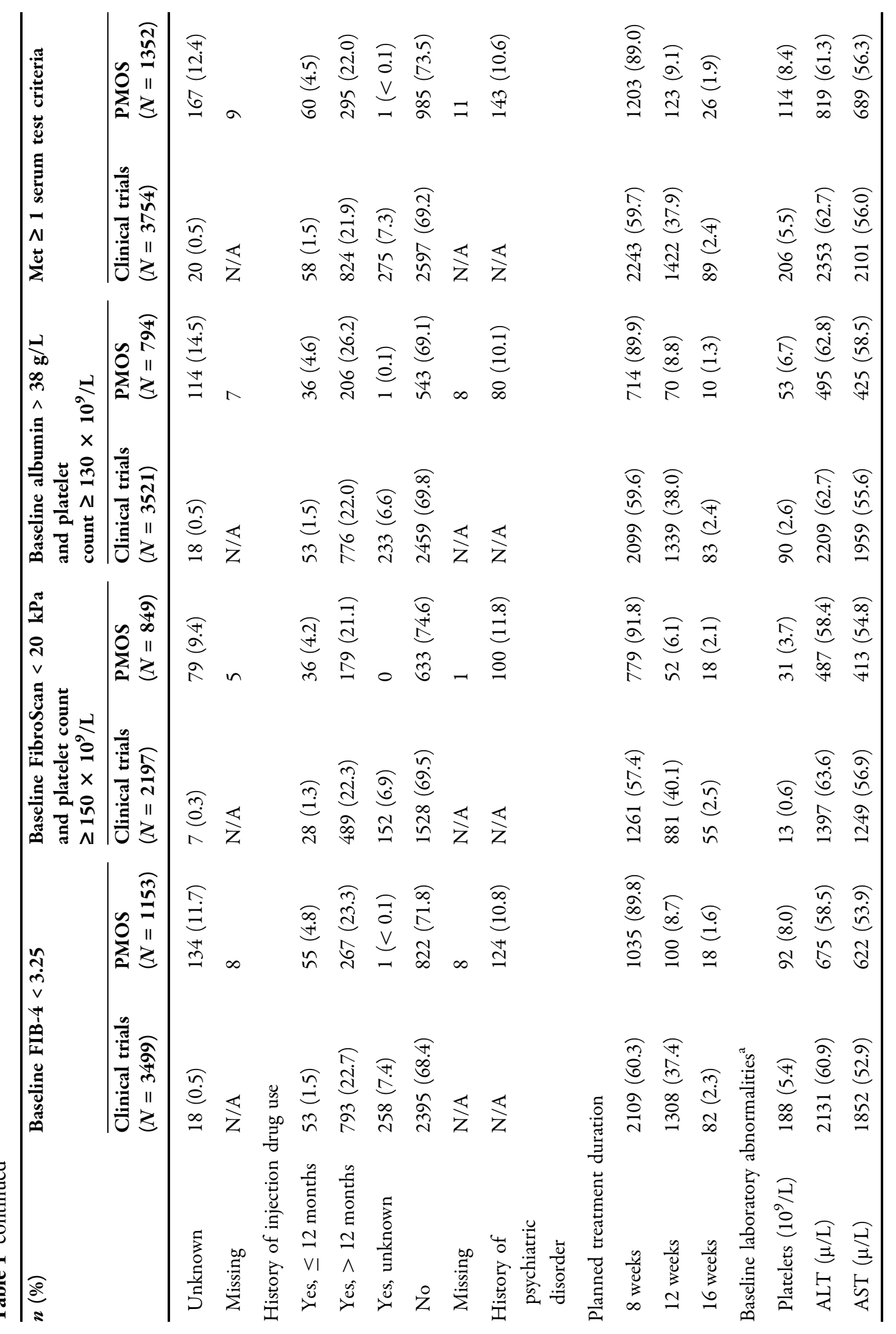




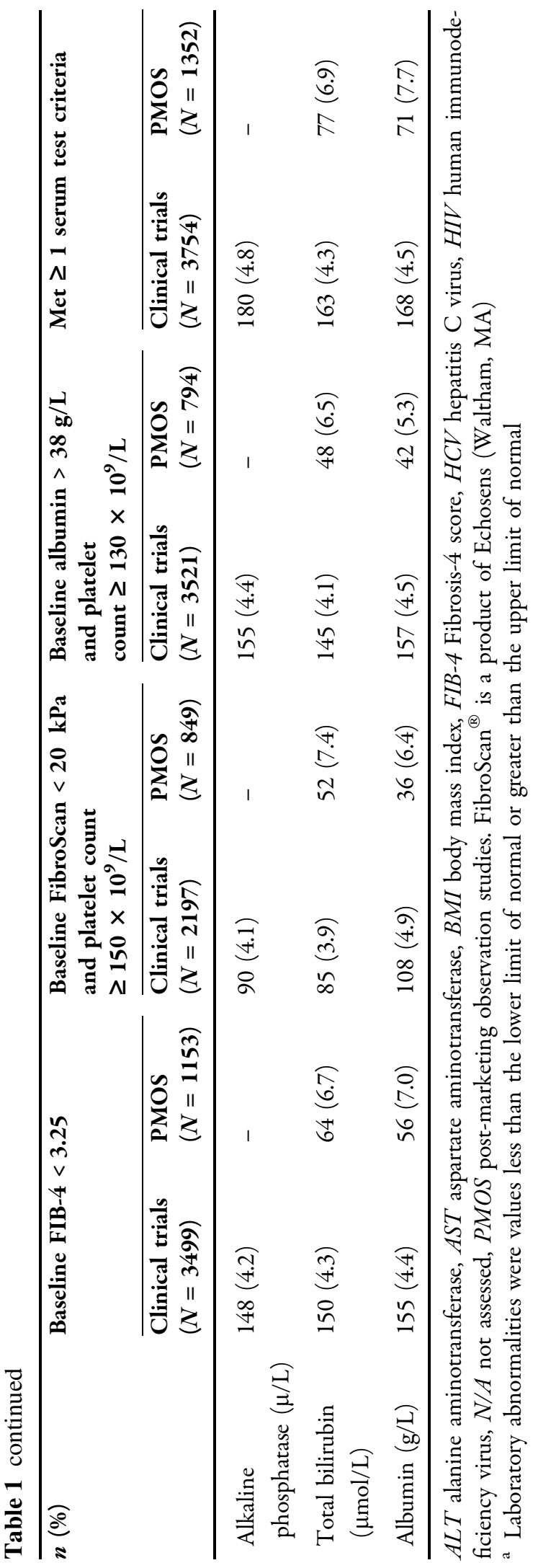

respectively. No serious AEs occurred in at least two patients in this cohort. Most common AEs were fatigue (34/1352 [2.5\%]), asthenia (33/ $1352[2.4 \%])$, and headache (30/1352 [2.2\%]). Most common AE leading to treatment discontinuation was nausea $(2 / 1352$ [0.1\%]). No patients in this cohort reported HCC or experienced treatment-emergent hepatic decompensation events.

When comparing safety data across the three analysis subgroups, no apparent differences in the number of AEs, treatment-related AEs, or serious AEs were observed within the clinical trial and PMOS cohorts. The most common AEs were consistent across subgroups in each cohort.

\section{Laboratory Assessments}

At baseline in the clinical trial cohort, $4.7 \%$ $(175 / 3754)$ of patients had thrombocytopenia (low platelets) and $0.1 \%(5 / 3754)$ had hypoalbuminemia (low albumin). There were $62.6 \%$ $(2351 / 3754), 56.0 \%(2101 / 3754), 4.1 \%$ (155/ $3754)$, and $3.1 \%(116 / 3754)$ of patients at baseline who had elevated ALT, AST, alkaline phosphatase, and total bilirubin, respectively. In the PMOS cohort at baseline, 6.1\% (83/1352) of patients had thrombocytopenia and $4.3 \%$ (40/926) had hypoalbuminemia. There were $61.0 \%(816 / 1337), 56.0 \%(685 / 1223)$, and $5.0 \%$ $(56 / 1116)$ of patients at baseline who had elevated ALT, AST, and bilirubin, respectively. Post-baseline grade 3 and 4 laboratory abnormalities were rare across all patient populations, and similar for the three unique subgroups. In the clinical trial cohort, $0.2 \%$ of patients experienced post-baseline grade $\geq 3$ ALT, AST, or bilirubin (Table 2). In the PMOS cohort, $0.2 \%$ and $0.1 \%$ of patients experienced post-baseline grade $\geq 3$ ALT and AST, respectively (Table 2). There were no post-baseline abnormalities for platelets or albumin. To assess the impact of G/P treatment on laboratory parameters, the change from baseline to maximum laboratory values during the treatment period was assessed to examine normalization of these parameters (Table 3). After treatment, the majority of patients in the clinical trial dataset had 


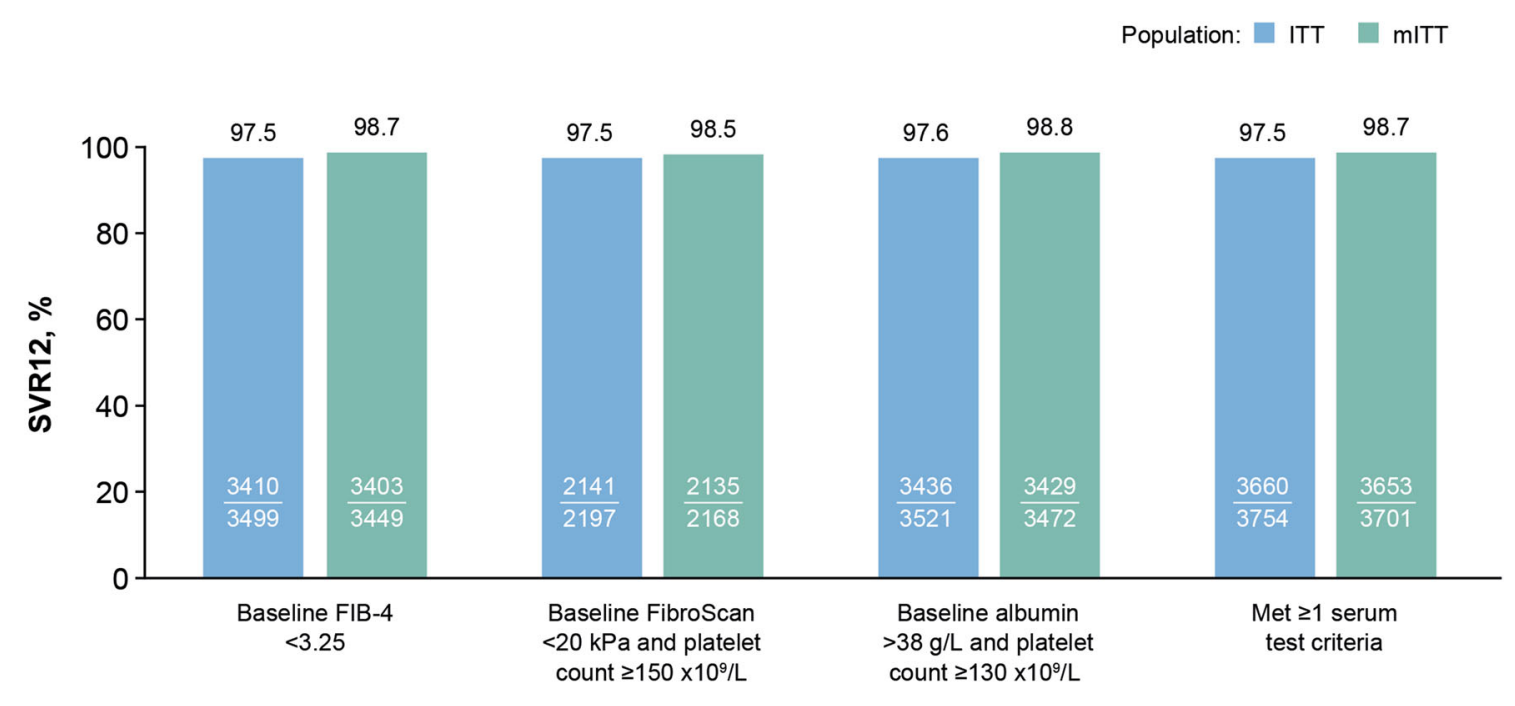

A Clinical trial cohort

Population: —ITT $\square$ CPSFU

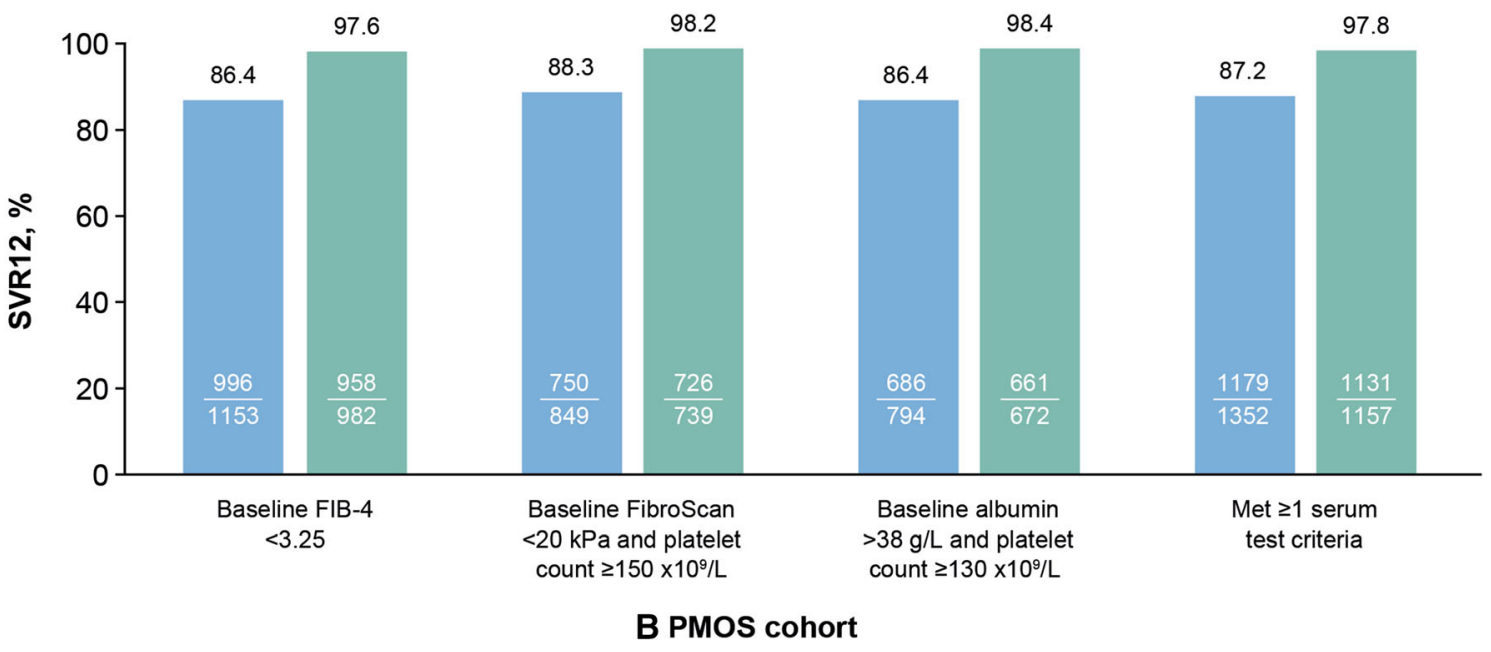

Fig. 1 SVR12 rates in the patient populations in the clinical trial cohort (a) and PMOS cohort (b). CPSFU population included patients from the core population, excluding those who did not have an HCV RNA evaluation after posttreatment day 70 for reasons not related to effectiveness or safety (lost to follow-up or unavailable HCV RNA data). Patients included in the CPSFU had one of the following: HCV RNA data after posttreatment day 70 (not included if the drug end date was unknown), virologic failure (on-treatment virologic failure or posttreatment relapse), discontinued the study because of an AE, and had HCV RNA $<50 \mathrm{IU} / \mathrm{mL}$ at the last measurement. mITT population excluded patients who did not achieve SVR for reasons other than virologic failure (e.g., patients who discontinued early or were lost to follow-up). AE adverse event, CPSFU core population with sufficient follow-up, FIB-4 Fibrosis-4 score, HCV hepatitis $\mathrm{C}$ virus, ITT intention-to-treat, mITT modified intention-to-treat, PMOS post-marketing observation studies, RNA ribonucleic acid, SVR12 sustained virologic response at posttreatment week 12. FibroScan ${ }^{\circledR}$ is a product of Echosens (Waltham, MA) 


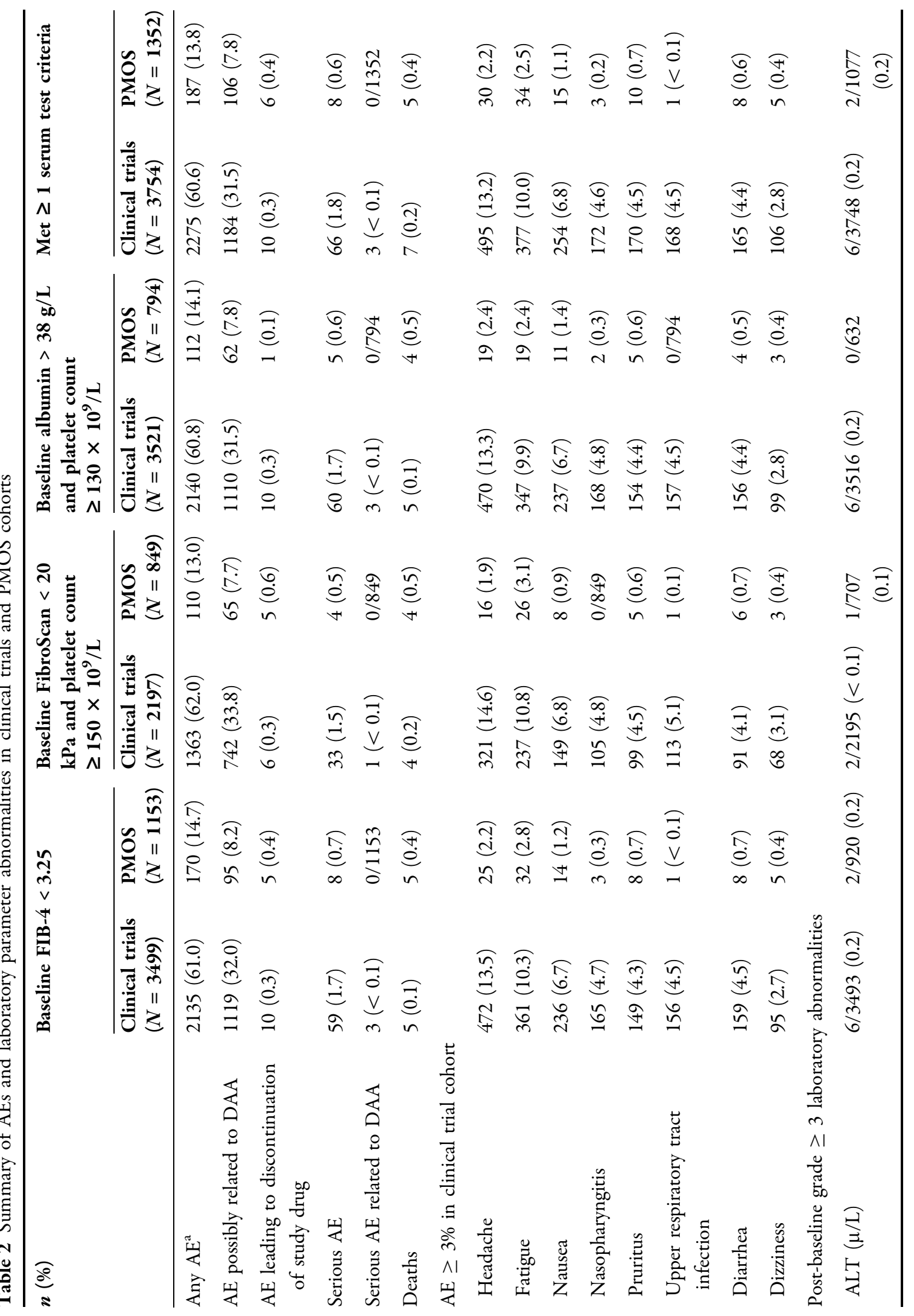




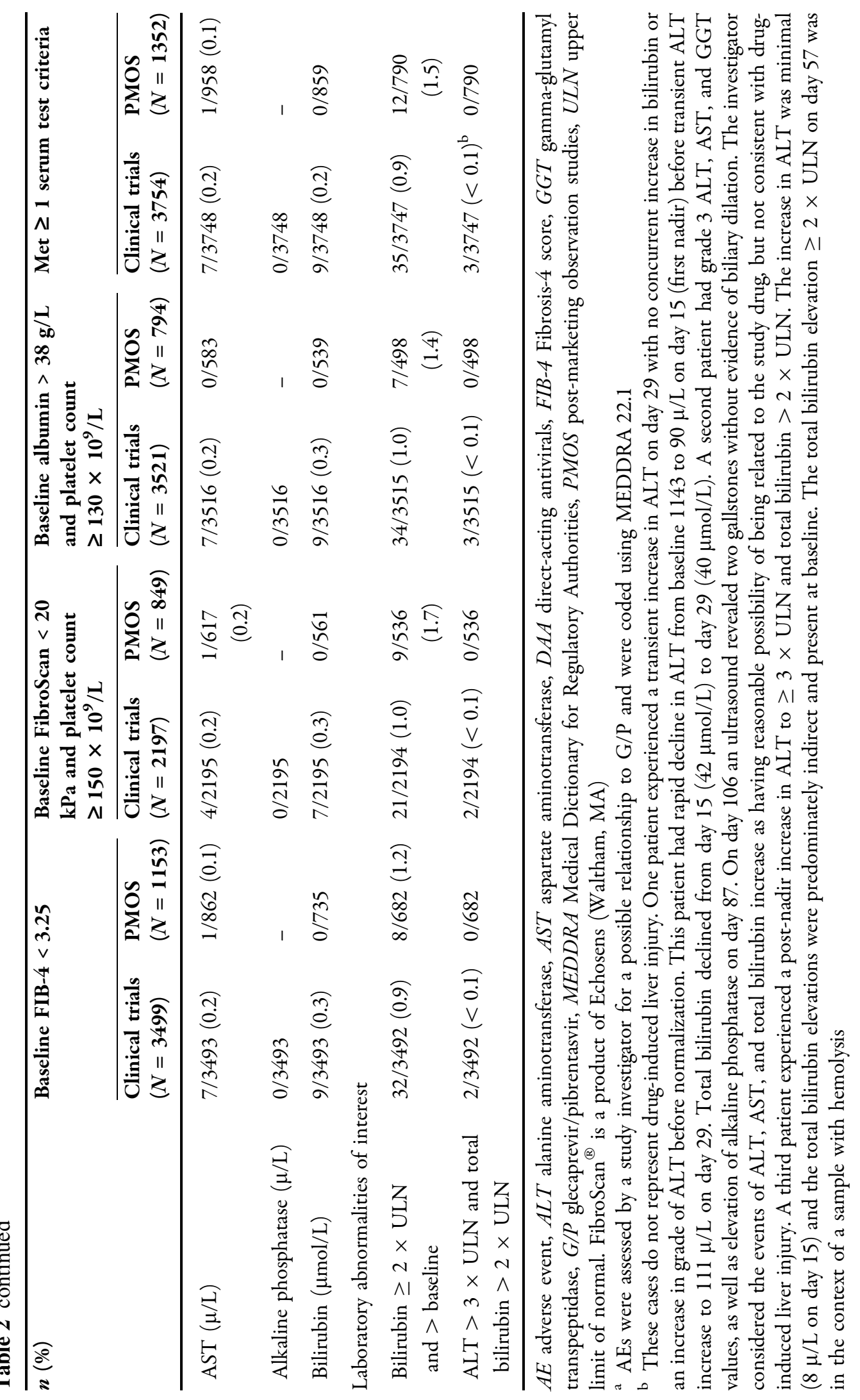


Table 3 Change in laboratory measures from baseline among patients who met at least one serum test criteria and with available data, $n / N(\%)$

\begin{tabular}{|c|c|c|}
\hline & Clinical trials $(N=3754)$ & $\operatorname{PMOS}(N=1352)$ \\
\hline \multicolumn{3}{|l|}{ Platelets $^{\mathrm{a}}$} \\
\hline Low to normal & $120 / 174(69.0)$ & $36 / 63(57.1)$ \\
\hline Normal to low & $15 / 3538(0.4)$ & $18 / 918(2.0)$ \\
\hline \multicolumn{3}{|c|}{ Alanine aminotransferase ${ }^{b}$} \\
\hline High to normal & $1549 / 2349(65.9)$ & $599 / 663(90.3)$ \\
\hline Normal to high & $34 / 1397(2.4)$ & $7 / 401(1.7)$ \\
\hline \multicolumn{3}{|c|}{ Aspartate aminotransferase ${ }^{c}$} \\
\hline High to normal & $1585 / 2100(75.5)$ & $456 / 513(88.9)$ \\
\hline Normal to high & $58 / 1648(3.5)$ & $9 / 406(2.2)$ \\
\hline \multicolumn{3}{|l|}{ Bilirubin $^{\mathrm{d}}$} \\
\hline High to normal & $21 / 116(18.1)$ & $12 / 40(30.0)$ \\
\hline Normal to high & $299 / 3585(8.3)$ & $56 / 746(7.5)$ \\
\hline \multicolumn{3}{|l|}{ Direct bilirubin $^{\mathrm{e}}$} \\
\hline High to normal & $63 / 204(30.9)$ & $24 / 65(36.9)$ \\
\hline Normal to high & $233 / 3176(7.3)$ & $36 / 209(17.2)$ \\
\hline \multicolumn{3}{|l|}{ Albumin ${ }^{\mathrm{f}}$} \\
\hline Low to normal & $4 / 5(80.0)$ & - \\
\hline Normal to low & $0 / 3580$ & - \\
\hline
\end{tabular}

PMOS post-marketing observation studies

${ }^{a}$ Lower limit of normal is 140

b Upper limit of normal is 32 for women and 43 for men

c Upper limit of normal is 34 for women and 36 for men

${ }^{\mathrm{d}}$ Upper limit of normal is $1.2 \mathrm{mg} / \mathrm{dL}$

e Upper limit of normal is $0.3 \mathrm{mg} / \mathrm{dL}$

${ }^{\mathrm{f}}$ Lower limit of normal is $33 \mathrm{~g} / \mathrm{L}$, data collection for albumin in the PMOS cohort was insufficient to report

normalized for platelets, ALT, and AST (69.0\%, $65.9 \%$, and $75.5 \%$, respectively), while $18.1 \%$, $30.9 \%$, and $80.0 \%$ of patients had normalized levels of bilirubin, direct bilirubin, and albumin, respectively (Table 3 ). Correspondingly, after treatment, the majority of patients in the PMOS dataset had normalized levels of platelets, ALT, and AST $(57.1 \%, 90.3 \%$, and $88.9 \%$, respectively), while $30.0 \%$ and $36.9 \%$ of patients had normalized levels of bilirubin and direct bilirubin, respectively (Table 3 ).

\section{DISCUSSION}

Revisions made to HCV testing algorithms and the advent of DAAs allow for the simplification of treatment for patients with $\mathrm{HCV}[24,25]$. Screening and treatment recommendations have expanded to be more inclusive, and in some cases recommend non-liver specialist treatment in low-risk patients with HCV, which represent the majority of patients [10]. This will increase treatment capacity, which may help to 
achieve the WHO's 2030 HCV elimination targets [26]. Some patients with advanced liver disease may require specialist care, including patients at risk for decompensation events and HCC [10]. Published data have begun demonstrating that non-liver specialist treatment of HCV-infected patients with DAAs can be as effective as specialist care, provided that nonspecialists receive appropriate training in the screening and treatment of HCV infection. The ASCEND study showed that task shifting HCV treatment to non-liver specialists was effective and well tolerated with no significant difference in SVR rate [27]. These results are supported by multiple studies that show HCV treatment through primary care is effective and may increase treatment uptake [28-30]. Despite these early data from non-liver specialist settings, concerns regarding safe use of $\mathrm{HCV}$ medications may remain, creating barriers for non-liver specialist HCV treatment uptake [31].

Data presented here describe the safety of G/P in low-risk patients with HCV identified by noninvasive techniques from both the clinical trial and PMOS cohorts. Overall, G/P was well tolerated in both cohorts with few patients experiencing AEs leading to treatment discontinuation $(0.3 \%$ and $0.4 \%$ in the clinical trial cohort and PMOS cohort, respectively), low rates of serious AEs $(1.8 \%$ and $0.6 \%$, respectively), and no significant hepatotoxicity observed. Fewer patients in the PMOS cohort had compensated cirrhosis compared with the clinical trial cohort. This difference could explain the higher number of decompensation events and patients with HCC observed in the clinical trial cohort. Safety data from this large analysis provide additional evidence for nonspecialist treatment following pretreatment assessment to identify low-risk patients through noninvasive diagnostics described by the three unique subgroups. Data derived from PMOS cohorts reinforce results that may be expected outside of clinical trial protocols; however, alone they may be limited by the underreporting of safety events typically present in observational studies versus more controlled clinical trials.

Comparisons between demographics and baseline characteristics of patients from the clinical trial and PMOS cohorts should be treated with caution because those in the clinical trials may not accurately represent real-world populations in regard to proportions with specific comorbidities or treatment durations studied. In this particular instance, fewer patients were administered 8-week G/P in the clinical trial cohort as a result of clinical trial designs aiming to identify optimal treatment duration across different patient subpopulations. However, G/P safety has been demonstrated to be similar regardless of treatment duration $[19,20]$.

Each noninvasive measure assessed here demonstrated a similar safety profile for G/P. The overlap and similar safety profile observed in patient subgroups qualified for the analysis by FIB- $4<3.25$ or albumin $>38 \mathrm{~g} / \mathrm{L}$ and platelet count $\geq 130 \times 10^{9} \mathrm{~L}$ suggest that either assessment would be sufficient to identify patients at risk for liver-related outcomes. Similar safety profiles were also observed across the FibroScan subgroup, but applicability and significance of this finding may be limited by lack of universal non-liver specialist access to this instrument.

A limitation to consider for FIB-4 eligibility is the finding of one patient in the clinical trial subgroup $(N=3499)$ who had baseline Child-Pugh B decompensated cirrhosis and experienced worsening of ascites. Despite being a protocol violation, this patient would have qualified for simplified treatment by FIB-4 in clinical practice, but not by the other two assessments. This may be explained because FIB-4 does not assess liver stiffness or symptoms of portal hypertension like the other two test criteria do, and relies more heavily on AST in its calculation, which was within the normal range for this patient. While not validated and perhaps a limitation, our observation of no hepatic decompensation events in patients identified by albumin $>38 \mathrm{~g} / \mathrm{L}$ and platelet count $\geq 130 \times$ $10^{9} / \mathrm{L}$ criteria is consistent with the publication first describing these criteria. In that analysis, no patient with CC experienced hepatic decompensation events during $G / P$ treatment [32]. Other limitations to consider are short duration of post-SVR monitoring for negative liver outcomes, missing baseline laboratory 
values in the PMOS cohort, and nonmutual exclusiveness of the subgroups meaning it is not possible to quantify how many of the criteria each patient met. A further limitation of this analysis is that in both clinical trial and PMOS cohorts, patients were assessed and treated by HCV specialists; more information is needed to confirm whether non-specialists would accurately identify patients with advanced fibrosis and both compensated and decompensated cirrhosis.

\section{CONCLUSIONS}

G/P treatment was well tolerated across the subgroups, including some patients with CC, consistent with pivotal clinical trial safety data [33] and real-world evidence [34] of G/P treatment in patients with CC. These data should provide reassurance that specialist intervention is not necessary for low-risk patients and reinforce the wider adoption of noninvasive screening tools in primary care settings. Nonliver specialists can be reassured that $G / P$, when prescribed per label, can be safely used in patients in combination with post-SVR HCC screening and awareness of potential drug-drug interactions $[10,35,36]$. In addition, these data may impact treatment guidelines, particularly in countries adopting decentralized HCV care, working towards simplified 8-week treatment for the majority of patients with HCV. These results may provide clinical confidence to physicians and other non-liver specialists treating HCV with the opportunity to expand the treater pool, a necessary step to meet elimination targets [31].

\section{ACKNOWLEDGEMENTS}

We thank the participants of these studies. Glecaprevir was identified by AbbVie and Enanta.

Funding. AbbVie funded this study and participated in the study design, research, analysis, data collection, interpretation of data, reviewing, and approval of the publication. AbbVie funded the journal's Rapid Service and Open Access of this publication.

Medical Writing Assistance. Medical writing support was provided by Annie Massa, MBiolSci, and Tom Owen, PhD, of Fishawack Communications, Ltd; and funded by AbbVie.

Authorship. All named authors meet the International Committee of Medical Journal Editors (ICMJE) criteria for authorship for this article, take responsibility for the integrity of the work as a whole, and have given their approval for this version to be published. All authors had access to relevant data and participated in the drafting, review, and approval of this publication. No honoraria or payments were made for authorship.

Authors' Contributions. SP, KC, JH, JH, PL, $\mathrm{AB}$, and SCG contributed to the collection of data, drafting and editing of the manuscript. $\mathrm{XF}, \mathrm{JJF}, \mathrm{MB}, \mathrm{MB}, \mathrm{JM}$, and IJ contributed to the analysis concept and methodology, collection of data, drafting and editing of the manuscript. DED, FT, and JM contributed to the analysis concept and methodology, drafting and editing of the manuscript. $\mathrm{AE}$ and $\mathrm{ZZ}$ contributed to analysis methodology, statistical analysis, drafting and editing of the manuscript.

Disclosures. Xavier Forns: Honoraria for consulting/speaking: AbbVie and Gilead. Jordan J. Feld: Research support/consultant: AbbVie, Arbutus, Eiger, Enanta, Gilead, GSK, Janssen, and Roche. Stanislas Pol: has received consulting and lecturing fees from Janssen, Gilead, MSD, AbbVie, Biotest, Shinogui, Roche, ViiV and grants from Gilead, Roche, and MSD. Kazuaki Chayama: Honoraria for teaching: AbbVie, Bristol-Myers Squibb, Dainippon Sumitomo, Eizai, Gilead, Mitsubishi Tanabe, MSD, and Otsuka; research funding from AbbVie and Bristol-Myers Squibb. Jinlin Hou: Consulting fees from AbbVie, Arbutus Biopharma, Bristol-Myers Squibb, Gilead Sciences, Johnson \& Johnson, and Roche; grants from Bristol-Myers Squibb and Johnson \& Johnson. Douglas E. Dylla, Mark Bondin, Fernando Tatsch, Margaret 
Burroughs, John Marcinak, Zhenzhen Zhang, Amanda Emmett: Employees of AbbVie and may hold stock/share options. Jeong Heo: Research support from Roche; advisor for AbbVie, Bristol-Myers Squibb, and Gilead Sciences. Pietro Lampertico: Speaker bureau and/or advisory board: AbbVie, Alnylam, Arrowhead, Bristol-Myers Squibb, Eiger BioPharmaceuticals, Gilead Sciences, GSK, Janssen, MSD, MYR Pharmaceuticals, Roche, and Spring Bank. Ashley Brown: Investigator, speaker honoraria, and advisor: AbbVie. Stuart C. Gordon: Grant/research support: AbbVie, Gilead, and Merck. Ira M Jacobson: Grant/research support: Assembly Biosciences, Bristol-Myers Squibb, Durect, Eli Lilly, Enanta, Gilead, Genfit, and Janssen; consultant/advisor: AbbVie, Arbutus Arrowhead, Atea, Assembly Biosciences, Bristol-Myers Squibb, Intercept, Janssen, Gilead, GSK, Merck, Novo Nordisk, Redhill.

Compliance with Ethics Guidelines. For all included studies, written informed consent was obtained from each patient, including consent to participate and permission to publish personal health information while maintaining the identity of the individual confidential. For all included studies, the study protocols conformed to the ethical guidelines of the 1964 Declaration of Helsinki and its later amendments, and were approved by the appropriate institutional review boards.

Data Availability. This clinical trial data can be requested by any qualified researchers who engage in rigorous, independent scientific research, and will be provided following review and approval of a research proposal and Statistical Analysis Plan (SAP) and execution of a Data Sharing Agreement (DSA). Data requests can be submitted at any time and the data will be accessible for 12 months, with possible extensions considered. For more information on the process, or to submit a request, visit the following link: https://www.abbvie.com/ourscience/clinical-trials/clinical-trials-data-andinformation-sharing/data-and-informationsharing-with-qualified-researchers.html.
Open Access. This article is licensed under a Creative Commons Attribution-NonCommercial 4.0 International License, which permits any non-commercial use, sharing, adaptation, distribution and reproduction in any medium or format, as long as you give appropriate credit to the original author(s) and the source, provide a link to the Creative Commons licence, and indicate if changes were made. The images or other third party material in this article are included in the article's Creative Commons licence, unless indicated otherwise in a credit line to the material. If material is not included in the article's Creative Commons licence and your intended use is not permitted by statutory regulation or exceeds the permitted use, you will need to obtain permission directly from the copyright holder. To view a copy of this licence, visit http:// creativecommons.org/licenses/by-nc/4.0/.

\section{REFERENCES}

1. European Association for the Study of the Liver. EASL recommendations on treatment of hepatitis C: final update of the series. J Hepatol. 2020;73: 1170-218.

2. Janjua NZ, Wong S, Darvishian M, et al. The impact of SVR from direct-acting antiviral- and interferonbased treatments for HCV on hepatocellular carcinoma risk. J Viral Hepat. 2020;27:781-793.

3. Ioannou GN, Green PK, Berry K. HCV eradication induced by direct-acting antiviral agents reduces the risk of hepatocellular carcinoma. J Hepatol. 2017;68:25-32. https://doi.org/10.1016/j.jhep. 2017.08.030.

4. Carrat F, Fontaine H, Dorival C, et al. Clinical outcomes in patients with chronic hepatitis $\mathrm{C}$ after direct-acting antiviral treatment: a prospective cohort study. Lancet. 2019;393:1453-64.

5. Kuna L, Jakab J, Smolic R, Wu GY, Smolic M. HCV extrahepatic manifestations. J Clin Transl Hepatol. 2019;7:172-82.

6. Cacoub P, Comarmond C, Domont F, Savey L, Desbois AC, Saadoun D. Extrahepatic manifestations of chronic hepatitis $C$ virus infection. Ther Adv Infect Dis. 2016;3:3-14. 
7. World Health Organization. Guidelines for the screening, care and treatment of persons with chronic hepatitis C infection; 2016. https://apps. who.int/iris/bitstream/handle/10665/205035/9789 241549615_eng.pdf;jsessionid=8D2F71004784EEC 3AD8BBDF63D13D49A?sequence $=1$. Accessed May 2020.

8. Alcorn K. France switches to simplified pangenotypic hepatitis C treatment; 2019. https://www. worldhepatitisalliance.org/latest-news/infohep/ 3435331/france-switches-simplified-pangenotypichepatitis-c-treatment. Accessed Mar 2020.

9. Gastroenterological Society of Australia. Australian recommendations for the management of hepatitis C virus infection: a consensus statement; 2018. https://www.hepcguidelines.org.au. Accessed Mar 2020.

10. AASLD-IDSA Hepatitis C Guidance Panel. Hepatitis C guidance 2019 update: American Association for the Study of Liver Diseases-Infectious Diseases Society of America recommendations for testing, managing, and treating hepatitis $\mathrm{C}$ virus infection. J Hepatol. 2019;2019(71):686-721.

11. Huppe D, Serfert Y, Buggisch P, et al. [4 years of direct-acting antivirals (DAAs) in the German Hepatitis C-Registry (DHC-R)]. Z Gastroenterol. 2019;57:27-36.

12. Radley A, Robinson E, Aspinall EJ, Angus K, Tan L, Dillon JF. A systematic review and meta-analysis of community and primary-care-based hepatitis C testing and treatment services that employ direct acting antiviral drug treatments. BMC Health Serv Res. 2019;19:765.

13. Crespo J, Albillos A, Buti M, et al. Elimination of hepatitis C. Positioning document of the Spanish Association for the Study of the Liver (AEEH). Gastroenterol Hepatol. 2019;42:579-92.

14. de Franchis R, Baveno VI Faculty. Expanding consensus in portal hypertension: report of the Baveno VI Consensus Workshop: stratifying risk and individualizing care for portal hypertension. J Hepatol. 2015;63:743-52.

15. Carvalho JR, Verdelho Machado M. New insights about albumin and liver disease. Ann Hepatol. 2018;17:547-60.

16. Bloom S, Kemp W, Nicoll A, et al. Liver stiffness measurement in the primary care setting detects high rates of advanced fibrosis and predicts liverrelated events in hepatitis C. J Hepatol. 2018;69: 575-83.

17. Srivastava A, Gailer R, Tanwar S, et al. Prospective evaluation of a primary care referral pathway for patients with non-alcoholic fatty liver disease. J Hepatol. 2019;71:371-8.

18. Kapadia SN, Marks KM. Hepatitis C management simplification from test to cure: a framework for primary care providers. Clin Ther. 2018;40: 1234-45.

19. AbbVie. Maviret (glecaprevir/pibrentasvir) summary of product characteristics; 2017. https://www. ema.europa.eu/en/documents/product-information/ maviret-epar-product-information_en.pdf. Accessed Mar 2020.

20. AbbVie. Mavyret (glecaprevir/pibrentasvir) US prescribing information; 2017. https://www. accessdata.fda.gov/drugsatfda_docs/label/2019/ 209394s008lbl.pdf. Accessed Mar 2020.

21. Zuckerman E, Gutierrez JA, Dylla DE, et al. Eight weeks treatment with glecaprevir/pibrentasvir is safe and efficacious in an integrated analysis of treatment-naive patients with hepatitis $\mathrm{C}$ virus infection. Clin Gastroenterol Hepatol. 2020;18: 2544-53.e6.

22. Panel A-IHCG. Recommendations for testing, managing, and treating hepatitis C; 2020. https:// www.hcvguidelines.org/. Accessed Mar 2020.

23. MedDRA. Introductory guide MedDRA version 22 . 1; 2019. https://admin.new.meddra.org/sites/ default/files/guidance/file/000354_intguide_22.1. pdf. Accessed June 2020.

24. Zoratti MJ, Siddiqua A, Morassut RE, et al. Pangenotypic direct acting antivirals for the treatment of chronic hepatitis $C$ virus infection: a systematic literature review and meta-analysis. EClinicalMedicine. 2020;18:

25. Peeling RW, Boeras DI, Marinucci F, Easterbrook P. The future of viral hepatitis testing: innovations in testing technologies and approaches. BMC Infect Dis. $2017 ; 17: 699$.

26. Sbarigia U, Wirth D, Van Nuys K, et al. Economic study of the value of expanding $\mathrm{HCV}$ treatment capacity in Germany. BMJ Open Gastroenterol. 2017;4:

27. Kattakuzhy S, Gross C, Emmanuel B, et al. Expansion of treatment for hepatitis $C$ virus infection by task shifting to community-based nonspecialist providers: a nonrandomized clinical trial. Ann Intern Med. 2017;167:311-8.

28. Wade A, Doyle J, Gane E, et al. Preliminary analysis of the Prime study; a randomized controlled trial comparing the hepatitis $\mathrm{C}$ care cascade in primary vs tertiary care. J Hepatol. 2018;68:S315-6. 
29. Syed TA, Bashir MH, Farooqui SM, et al. Treatment outcomes of hepatitis C-infected patients in specialty clinic vs. primary care physician clinic: a comparative analysis. Gastroenterol Res Pract. 2019;2019:8434602.

30. Norton BL, Fleming J, Bachhuber MA, et al. High HCV cure rates for people who use drugs treated with direct acting antiviral therapy at an urban primary care clinic. Int J Drug Policy. 2017;47: 196-201.

31. Tran TT. Hepatitis C: who should treat hepatitis C virus? The role of the primary care provider. Clin Liver Dis (Hoboken). 2018;11:66-8.

32. Krassenburg L, Mann R, Ramji A, et al. Identification of patients with compensated cirrhosis who can safely use protease inhibitor-based therapy for HCV infection. J Hepatol. 2020;73(suppl1): S349-S350.
33. Brown RS Jr, Buti M, Rodrigues L, et al. Glecaprevir/ pibrentasvir for 8 weeks in treatment-naive patients with chronic HCV genotypes 1-6 and compensated cirrhosis: the EXPEDITION-8 trial. J Hepatol. 2020;72:441-9.

34. Lampertico P, Mauss S, Persico M, et al. Real-world clinical practice use of 8-week glecaprevir/pibrentasvir in treatment-naive patients with compensated cirrhosis. Adv Ther. 2020;37:4033-42.

35. Carmona I, Cordero P, Ampuero J, Rojas A, RomeroGomez M. Role of assessing liver fibrosis in management of chronic hepatitis $\mathrm{C}$ virus infection. Clin Microbiol Infect. 2016;22:839-45.

36. Schulte B, Wubbolding M, Marra F, et al. Frequency of potential drug-drug interactions in the changing field of HCV therapy. Open Forum Infect Dis. 2020;7:ofaa040. 